Fazit: Frauenthemen sind zeitlos - trotz scheinbar unterschiedlicher Lebenswirklichkeiten. Damit es weitergehen kann: Sprecht die Älteren an.
Der 38. FJT wird im Mai 2012 in Bremen stattfinden. Die Inhalts- und die Organisationsgruppe suchen jeweils noch dringend Verstärkung!

\title{
Der djb geht zu Hauptversammlungen: Ein Erfahrungsbericht
}

\author{
Kivilcim Simsek \\ Mitarbeiterin des djb-Projekts „Aktionärinnen fordern Gleichbe- \\ rechtigung“; Rechtsanwältin, Berlin
}

Auch 2011 hat sich der djb auf die Fahne geschrieben, von den großen DAX, MDAX und TecDAX Unternehmen so viele wie möglich durch das Fragerecht dank Aktienbesitz bei den Hauptversammlungen auf das Thema Frauen in Führungspositionen anzusprechen. 75 Unternehmen wurden von vielen ehrenamtlichen Teilnehmerinnen und zwei Projektmitarbeiterinnen - eine davon ich - und in den unterschiedlichsten Städten Deutschlands besucht. In den wenigen Wochen lernte ich so die verschiedensten Unternehmen, Versammlungsorte und Bahnhöfe kennen.

Obwohl der Ablauf im Wesentlichen immer gleich war, stellte jede Hauptversammlung auch individuelle Anforderungen an mich als Rednerin. Die gefüllte Messe-Halle in Frankfurt oder die Olympiahalle in München forderten den Schneid, unbequeme Fragen vor 5.000 und mehr Menschen zu stellen. An anderen Tagen stellten Kleinaktionäre im Anschluss an die Redebeiträge unbequeme Fragen an mich. Lob und positive Resonanzen motivierten aber auch immer, das Thema voranzutreiben.

Der Besuch einer Hauptversammlung beginnt mit der Anmeldung der Fragen des djb, die sechs Tage zuvor per Post an die jeweiligen Unternehmen geschickt wurden. Daher waren alle Unternehmen, die wir besucht haben, bereits über unsere Teilnahme informiert. Zur Sicherheit reichten wir unsere Fragen regelmäßig am Versammlungstag noch einmal am Wortmeldetisch mit der Bitte um detaillierte Beantwortung ein.

Sodann begann jede Hauptversammlung mit einem Lagebericht des Aufsichtsratsvorsitzenden, der anschließend das Wort dem Vorstandsvorsitzenden übergab. Dieser berichtete von dem abgelaufenen Geschäftsjahr und über die Ziele des bevorstehenden Geschäftsjahrs. Teilweise kam es vor, dass der Aufsichtsratsvorsitzende oder der Vorstandsvorsitzende bereits dort das Thema „Frauen in Führungspositionen“ ansprachen. Allerdings gingen diese Bemerkungen meist nicht über das bereits im Geschäftsbericht Veröffentlichte hinaus. Vorwiegend teilten sie flüchtig mit, ob sie den Empfehlungen des Corporate Governance Kodexes entsprochen haben oder nicht. Unter großer Zustimmung im Publikum betonten manche auch kurz, dass sie gegen eine Frau- enquote seien oder behaupteten oberflächlich, dass es im Interesse des Unternehmens nur auf die Qualifikation, Erfahrungen und Kompetenzen der Kandidatinnen und Kandidaten ankäme.

Das Auditorium setzte sich neben den professionellen Aktionärsvertreterinnen und -vertretern meist aus älteren Aktionärinnen und Aktionären, Rentnerinnen und Rentnern zusammen. Neben dem Interesse am Unternehmen schenkten diese auch dem kostenfreien Mittagessen ihre Aufmerksamkeit und verließen den Saal pünktlich zur Buffeteröffnung. Ich kann nur raten, ob es Zufall war, dass unsere Redebeiträge meist zu dieser Mittagszeit stattfanden. Nach über 20 Versammlungen schienen viele der Aktionärinnen und Aktionäre, die ebenfalls häufig Versammlungen besuchten, schon fast klassisch nach Pawlow konditioniert bei der Nennung unserer Namen zu reagieren; des Öfteren standen Aktionärinnen und Aktionäre mit Redebeginn auf und verließen den Saal. Ebenfalls fielen gelegentlich abfällige Bemerkungen, auch von Aktionärinnen.

Dennoch bekamen wir nach unserem Wortbeitrag zumeist zustimmenden Beifall, und auch Szenenapplaus hat es gegeben. Auf der Hauptversammlung der ElringKlinger AG in Stuttgart bekamen wir allerdings gar keinen Beifall. Als zweite Teilnehmerin war ich die Einzige im Saal, die unserem Wortbeitrag applaudierte.

In der Regel wurden die Hauptversammlungen zu zweit besucht, meist waren es ein ehrenamtliches Mitglied des djb, unterstützt insbesondere bei der Protokollführung durch eine Projektmitarbeiterin.

Während die erste Teilnehmerin Fragen zu den Empfehlungen des Corporate Governance Kodexes stellte, waren die Fragen der zweiten Teilnehmerin auf konkrete Zahlen und Fakten hinsichtlich der Frauenbeteiligung im Unternehmen gerichtet. Sofern ich die Hauptversammlungen alleine besuchte, wurden beide Wortbeiträge zu einem zusammengefügt.

Bei den meisten Versammlungen wurden die Redebeiträge auf Leinwände und Fernseher übertragen. So wurde sichergestellt, dass nicht nur im Veranstaltungssaal selbst, sondern auch am Buffet und akustisch sogar auf den Toiletten den Wortbeiträgen gelauscht werden konnte. Es war nicht möglich uns nicht zu hören. Auch wenn manche den Saal verließen, weil wir in der Rednerliste weit hinten standen oder das Mittagessen rief, waren wir sicher, dass die Anwesenden das Thema hörten. 
Einige Unternehmen beantworteten unsere Fragen sehr ausführlich. Es war zu beobachten, dass sie das Thema „Frauen“ sehr ernst nahmen. Andere Unternehmen gingen nur oberflächlich auf die Fragen ein, beantworteten sie teilweise gar nicht oder ratterten ganz hastig die Antworten herunter, was das Protokollieren sehr erschwerte.

Die Rhön-Klinikum AG wollte sich nur widerwillig mit uns auseinandersetzen. Trotz mehrfacher Bitten um Beantwortung der Fragen der ersten Rednerin wies man meine Projektkollegin Sarah Nußbaum darauf hin, sie könne gegen die Hauptversammlung Widerspruch einlegen, wenn sie mit dem bisher Gesagten nicht zufrieden sei.

Sofern unsere Fragen nicht beantwortet wurden, meldeten wir unsere Fragen ein zweites Mal an und wurden erneut ans Rednerpult gerufen. Teilweise war es uns auch möglich an den Notar heranzutreten und diesen darauf aufmerksam zu machen, dass unsere Fragen nicht beantwortet wurden. Meist führte dies zur Beantwortung der noch offenen Fragen, so manches Mal blieben Unternehmen uns Antworten jedoch schuldig.

Im Anschluss an die Hauptversammlung oder während einer Pause für die Auswertung der Wahlen versuchten wir an eine Frau aus dem Aufsichtsrat und/oder Vorstand heranzutreten und unsere Broschüre vom Vorjahr „Aktionärinnen fordern Gleichberechtigung - Mehr Frauen in Führungspositionen 2010“ zu überreichen. Dabei folgte gelegentlich sogar ein persönliches Gespräch. Beispielsweise kam auf der Hauptversammlung der Celesio AG in Stuttgart, die ich mit Rechtsanwältin Annette Vees besuchte, Susan Naumann, Gewerkschaftssekretärin, ver.di - Vereinte Dienstleistungsgewerkschaft e. V. und Aufsichtsratsmitglied, zu uns. Sie lobte unser Engagement und bekam unsere Broschüre überreicht. Auch bei der Hauptversammlung der SolarWorld AG kam es zu einem ermutigenden Gespräch bei Kaffee und Kuchen zwischen Colette Rückert-Hennen, inzwischen Vorstand Personal und Marke/Chief Human Resources and Brand Officer (CHRBO) und EIGE-Programmdirektorin (EIGE: European Institute for Gender Equality) Barbara Wurster sowie Sarah Nußbaum, den beiden djb-Vertreterinnen.

Wir versuchten auf den Hauptversammlungen auch, unsere djb-Flugblätter zur Aktion „Hauptversammlungen“ zu verteilen, um viel Öffentlichkeit zu erreichen. Da das Verteilen der Flyer meist untersagt war, baten wir die Damen am Informations- bzw. Pressestand, unsere Flyer an die Presseleute weiter zu geben.

Teilweise wurde ich im Anschluss an die Veranstaltungen von Aktionärinnen und Aktionären persönlich angesprochen, die unser Projekt befürworteten und es sehr lobten, dass wir uns für die Frauen so sehr einsetzten. So viele Befürwortungen es gab, gab es aber leider auch negative Anmerkungen, wie „kompetente Frauen brauchen nicht die Frauenquote, da diese es schaffen, sich durchzusetzen“ oder „die Frauenquote ist verfassungswidrig und verstößt gegen Artikel 3 des Grundgesetzes, weil allen Bewerbern die gleichen Chancen zustehen müssen“ und sehr oft kam die Bemerkung „der Deutsche Ju- ristinnenbund ist selbst diskriminierend, weil Männer dort noch nicht einmal Mitglieder werden können ...". Sehr erstaunlich war es allerdings, dass die Zustimmung und die Motivation häufig von Männern kamen. Umso enttäuschter musste ich feststellen, dass einige Frauen uns wenig unterstützten. So rüttelte eine ältere Dame mit Beginn unseres Wortbeitrags auf der Hauptversammlung der ProSiebenSat.1 Media AG ihren Ehemann so lange, bis dieser mit ihr den Saal verließ.

Auf einigen Hauptversammlungen wurde das Thema auch von anderen Rednern während der Generaldebatte angesprochen. Während manche die Erhöhung des Frauenanteils anstrebten, sprachen sich andere gegen eine Frauenquote aus. Fast jedes Unternehmen hat sich gegen jede Art einer gesetzlichen Quote, insbesondere gegen eine starre Frauenquote, ausgesprochen. Viele Unternehmen begründeten diese Ansicht damit, dass eine gesetzliche Quote die Interessen des Unternehmens und eine qualitative Unternehmensführung außer Acht lasse. Die Unternehmen erklärten, dass man zwischen den Unternehmen unterscheiden müsse. Es sei einfacher, den Anteil der Frauen in einem Unternehmen, welches auf Mode, Pflege und Dienstleistungen gerichtet ist, zu erhöhen, während man bei Unternehmen, die vielmehr technisch ausgerichtet sind, kaum Frauen finde. Die meisten technischen Unternehmen stützten sich auf diese Schutzbehauptung und zeigten einen „angeblichen Willen“ den Frauenanteil im Unternehmen zu erhöhen, sofern die Anzahl der Absolventinnen in den technischen Studiengängen mehr werden sollte.

Die Erfahrungen aus den Besuchen der Hauptversammlungen zeigen, dass das Thema noch immer viele Gemüter erhitzt und noch viel Diskussionsbedarf besteht. Umso wichtiger scheint es mir, dass auch dieses Jahr wieder viele Hauptversammlungen besucht wurden und so viel Öffentlichkeit erreicht wurde.

Vor vielen Menschen für eine Sache einzutreten hat mich um viele persönliche Erfahrungen bereichert. Ich bin gespannt, zu welchen Ergebnissen die Auswertung der gesammelten Daten kommen wird und hoffe, mit meinem Einsatz dafür gesorgt zu haben, dass in den Führungsetagen der DAX-Unternehmen mehr über das Thema „Frauen in Führungspositionen“ gesprochen wird. Denn bereits jetzt lässt sich feststellen, dass eine Verbesserung zum Vorjahr eingetreten ist. So sind teilweise in den Geschäfts- und Nachhaltigkeitsberichten frauenspezifische Daten und Fakten ausführlich erhoben worden. Dies wurde mir auch von den Teilnehmerinnen und Teilnehmern, die unsere Aktion wiederholt unterstützten, bestätigt. Unsere Aktion „Aktionärinnen fordern Gleichberechtigung “ mag viel zu diesen Verbesserungen beigetragen haben.

Die Hauptversammlungsbesuche - insbesondere die Antworten der Aufsichtsrats- und Vorstandsmitglieder - wurden von den Teilnehmerinnen protokolliert und warten derzeit auf ihre Auswertung und Veröffentlichung in der Projektstudie 2011, die am 16. November 2011 im Europäischen Haus, Berlin präsentiert werden soll (s. Terminhinweis S. 148). 